\title{
Randomized Multicenter Evaluation of Quality of Life and Treatment Satisfaction in Type 2 Diabetes Patients Receiving Once-Weekly Trelagliptin Versus a Daily Dipeptidyl Peptidase-4 Inhibitor
}

\author{
Hitoshi Ishii · Yuki Suzaki · Yuko Miyata · Shingo Matsui (D)
}

Received: March 15, 2019 / Published online: June 18, 2019

(C) The Author(s) 2019

\begin{abstract}
Introduction: Dipeptidyl peptidase-4 (DPP-4) inhibitors are an established treatment in type 2 diabetes mellitus (T2DM). The objective of this study was to investigate differences in quality of life (QOL) and treatment satisfaction among treatment-naïve T2DM patients receiving onceweekly trelagliptin or a daily DPP-4 inhibitor.

Methods: In this multicenter, randomized, open-label, parallel-group, phase IV study conducted in Japan, 218 patients were randomized to trelagliptin $100 \mathrm{mg}$ once weekly or a once- or twice-daily DPP-4 inhibitor for 12 weeks (NCT03014479; JapicCTI-173482). QOL and treatment satisfaction were assessed using the Diabetes Therapy-Related QOL (DTR-QOL) Questionnaire and Diabetes Treatment
\end{abstract}

Enhanced Digital Features To view enhanced digital features for this article go to https://doi.org/10.6084/ m9.figshare.8192168.

Electronic Supplementary Material The online version of this article (https://doi.org/10.1007/s13300019-0643-1) contains supplementary material, which is available to authorized users.

H. Ishii

Department of Diabetology, Nara Medical

University, Nara, Japan

Y. Suzaki · Y. Miyata · S. Matsui (ه)

Japan Medical Affairs, Takeda Pharmaceutical

Company Limited, Tokyo, Japan

e-mail: shingo.matsui@takeda.com
Satisfaction Questionnaire (DTSQ), respectively. The primary endpoint was change from baseline in DTR-QOL total score at week 12. Secondary endpoints included further analysis of the DTRQOL and DTSQ components. Other endpoints included glycemic control, treatment adherence, and safety.

Results: The between-group difference in the change from baseline to week 12 in DTR-QOL total score was 2.418 (95\% confidence interval $-1.546,6.382 ; P=0.2305)$. Analysis of the DTR-QOL and DTSQ results by subscales and stratification generally showed a numerical improvement with trelagliptin over daily DPP-4 inhibitors. QOL and treatment satisfaction improved with a reduction in frequency of concurrent and study drug dosing. Treatment adherence was $>97 \%$ for both groups. The effect of trelagliptin on glycemic control was similar to that seen with daily DPP-4 inhibitors. Trelagliptin and daily DPP-4 inhibitors were well-tolerated and demonstrated similar safety profiles.

Conclusions: Once-weekly trelagliptin $100 \mathrm{mg}$ administered for 12 weeks resulted in a numerically, but not statistically, greater improvement in QOL and treatment satisfaction versus daily DPP-4 inhibitors. The decision to administer once-weekly or daily DPP-4 inhibitor treatment is likely to depend on patient preferences and the treatment policies of physicians.

Trial Registration: ClinicalTrials.gov (NCT03 014479) and JAPIC (JapicCTI-173482). 
Funding: Takeda Pharmaceutical Company Ltd.

Keywords: Adherence; DPP-4 inhibitors; DTRQOL; Quality of life; Treatment satisfaction; Trelagliptin; Type 2 diabetes

\section{INTRODUCTION}

Diabetes is a complex, chronic, and progressive metabolic disease requiring ongoing, multifactorial medical care $[1,2]$, with the ultimate aim of treatment being to maintain quality of life (QOL) and life expectancy at a level similar to those without diabetes [3]. Uncontrolled diabetes is associated with both acute, life-threatening consequences and long-term complications that substantially increase diabetes-related morbidity and mortality $[2,3]$. In addition, data suggest that patients with diabetes have a lower QOL than patients without diabetes [4-6], and that the presence of diabetes-related complications decreases QOL [7]. Well-controlled blood glucose, lipid levels, and blood pressure can prevent complications in patients with diabetes, which can subsequently lead to a positive impact on patient QOL and overall prognoses [3].

Despite the benefits associated with diabetes treatment, adherence to anti-diabetic medication among patients with diabetes is typically poor [8]. A major reason for this lack of adherence is treatment complexity; the chronic nature of diabetes means that treatment is lifelong, and the progressive nature of the disease in addition to its related complications means that medication regimens increase in complexity over time [8]. Studies have shown that patients with diabetes are more adherent to medications that are dosed less frequently [9-12], suggesting that a reduction in dosing frequency may increase treatment satisfaction and eliminate a barrier to treatment continuation.

Dipeptidyl peptidase-4 (DPP-4) inhibitors are a class of oral anti-diabetic agents that inhibit degradation of the incretin hormones glucosedependent insulinotropic polypeptide and glucagon-like peptide 1 by DPP-4, facilitating the improvement of glycemic control through prolonging the action of these hormones on insulin release, glucose responsiveness, and gastric emptying [13]. Sitagliptin was the first DPP-4 inhibitor approved in Japan in December 2009, followed by alogliptin in April 2010 [14]. Unlike the early DPP-4 inhibitors, which are administered once or twice daily, trelagliptin, which was approved for use in Japan in 2015 is administered once weekly $[13,15]$. A phase III study has shown that trelagliptin was non-inferior to once-daily alogliptin in lowering hemoglobin A1c (HbA1c) in Japanese patients with type 2 diabetes (T2DM) [15].

Based on the clinical trial results to date, trelagliptin is expected to be equally effective in patients with T2DM for whom a once-daily DPP-4 inhibitor is appropriate [16]. The objective of this randomized, multicenter study was to investigate differences in QOL and treatment satisfaction among treatment-naïve patients with T2DM receiving either trelagliptin or a daily DPP-4 inhibitor for 12 weeks.

\section{METHODS}

\section{Study Design}

This was a multicenter, randomized, open-label, parallel-group, phase IV study conducted in Japan between February and October 2017. As per the study protocol [17], patients were randomized (1:1) to trelagliptin $100 \mathrm{mg}$ once weekly or a daily DPP-4 inhibitor administered once or twice daily, as per the product label. Randomization was stratified by HbA1c $(<8.0 \%$ or $\geq 8.0 \%$ ) and total score for the Diabetes Therapy-Related Quality Of Life (DTR-QOL) Questionnaire $(<80$ or $\geq 80)$. The study consisted of a 4-week screening period and a 12 -week treatment period. Patients were assessed during screening at week -4 , and at weeks 0,4 , and 12 of the treatment period.

The study was conducted in compliance with the Japanese Ethical Guideline for Clinical Research, all applicable local regulations, and the ethical principles set out in the Declaration of Helsinki and the Good Clinical Practice consolidated guidelines. All regulation-specified documentation, including the study protocol and all revisions, was reviewed and approved by 
an independent ethics committee at each study site (as listed in the electronic supplementary material). All patients provided written informed consent before any screening procedures were undertaken. The study was conducted at 25 sites in Japan and is registered at ClinicalTrials.gov (NCT03014479) and JAPIC (JapicCTI-173482).

\section{Patient Population}

Patients aged $\geq 20$ years with T2DM were included in this study with $\mathrm{HbA} 1 \mathrm{c} \geq 6.5 \%$ and $<10.0 \%$ at the start of screening. Other inclusion criteria included: diabetes management consisting of diet and exercise only for at least 12 weeks prior to screening; requirement for DPP-4 inhibitor treatment; receiving $\leq 2$ concomitant medications for treatment of comorbidities; and completion of the DTR-QOL questionnaire at the start of the screening period (week - 4).

The main exclusion criteria were: a diagnosis of type 1 diabetes; receiving any oral antidiabetic medication at the start of the screening period; severe renal impairment or renal failure, i.e., estimated glomerular filtration rate $<30 \mathrm{~mL} / \mathrm{min} /$ $1.73 \mathrm{~m}^{2}$ or receiving dialysis; serious heart disease or cerebrovascular disorder; a serious pancreatic or hematologic disease, or any other disease; a history of gastrointestinal resection; the presence of proliferative diabetic retinopathy or malignant tumors; a history of hypersensitivity or allergy to DPP-4 inhibitors; or participation in other clinical studies. Pregnant or lactating women or women who were planning a pregnancy during the study period were excluded, as were patients who required a change in their concomitant medication regimen (addition, discontinuation, or dosage change), or who required treatment with a prohibited concomitant medication during the study period. Prohibited medication was defined as any glucose-lowering medication other than that allocated as part of the study.

\section{Study Endpoints}

The primary efficacy endpoint was the change from baseline (week 0) in DTR-QOL total score at week 12. Secondary efficacy endpoints included changes from baseline in the total score for each of the four factors included in the DTR-QOL questionnaire (factor 1: burden on social activities and daily activities; factor 2 : anxiety and dissatisfaction with treatment; factor 3: hypoglycemia; factor 4: treatment satisfaction); the change in the DTR-QOL total score at each assessment time point; and the change in the Diabetes Treatment Satisfaction Questionnaire (DTSQ) total score at each assessment time point. In addition, the change in the DTRQOL and DTSQ total scores, stratified by the following factors at week 0 , was also assessed: use of medication for the treatment of comorbidities; number of daily doses of medication for the treatment of concurrent comorbidities $(<2$ times or $\geq 2$ times); total number of daily tablets of medication for the treatment of concurrent comorbidities $(<2$ tablets or $\geq 2$ tablets); and number of doses of the onceweekly study drug trelagliptin (100 $\mathrm{mg})$, the once-daily comparator drugs sitagliptin ( $25 \mathrm{mg}$ or $50 \mathrm{mg}$ ), alogliptin (12.5 mg or $25 \mathrm{mg}$ ), linagliptin $(5 \mathrm{mg})$, teneligliptin $(20 \mathrm{mg})$ or saxagliptin $(5 \mathrm{mg})$, or the twice-daily comparator drugs vildagliptin $(50 \mathrm{mg})$ or anagliptin (100 mg).

Additional endpoints included laboratory tests (HbA1c, fasting blood glucose, glycoalbumin, 1,5-anhydroglucitol [1,5-AG]) and treatment adherence.

The safety endpoint was adverse events (AEs) occurring after the first administration of study drug or comparator. Serious AEs (SAEs) were defined as an $\mathrm{AE}$ that resulted in death, was life threatening, required inpatient hospitalization or prolongation of existing hospitalization, or resulted in persistent or significant disability or incapacity.

\section{Treatment Adherence}

To assess treatment adherence, study drug usage was recorded using an electronic medication record card (Your Manager ${ }^{\circledR}$; Dai Nippon Printing Co., Ltd., Japan). After the patients took their medication, they pressed the button on the medication record card, which recorded the 
date and time. Patients were instructed to bring empty press-through-package sheets to each visit, which were then assessed by the investigator for evidence of non-adherence.

\section{DTR-QOL and DTSQ}

Patients answered all 29 questions of the DTRQOL questionnaire [18] at all assessment time points. The DTR-QOL response scale used a 7-point Likert scale, where 1 indicated 'strongly agree' and 7 indicated 'strongly disagree' [18]; calculation of the total score per factor was achieved by totaling the item scores and converting them into a score between 0 and 100 , where the best-case response was 100 and the worst-case response was 0. For Factor 4, where the items were positive statements [18], the scores were reversed, added, and converted as above. Calculation of the total overall score was achieved by adding the scores for each of the 29 items and converting the score into a number between 0 and 100. Missing scores were handled as follows: for individual factors with $<50 \%$ missing data, the mean value calculated from available answers was applied to cover the missing data, while the total score was not calculated for factors with $\geq 50 \%$ missing data. For the overall score, the total score of complete questions was not calculated if a total score was not available for any of the four factors.

Patients answered all eight questions of the DTSQ $[19,20]$ at all assessment time points.

\section{Statistical Methods}

Planned enrolment for the study was 240 patients (120 patients in each treatment group), since a sample size of 110 patients per group was required to ensure $80 \%$ power for betweengroup comparisons [with assumed changes from baseline to end of study in the primary endpoint (DTR-QOL total score) based on published values for both treatment groups $[19,21]]$, assuming a discontinuation rate of $8 \%$ (based on a previous report [22]). Two analysis sets were defined for this study: the full analysis set (FAS), defined as all randomized patients who received at least one dose of study drug, and the safety analysis set (SAS), defined as all patients who received at least one dose of study drug. The FAS was used for all efficacy analyses and the SAS was used for all safety analyses.

Summary statistics, including the number of patients, mean, standard deviation (SD), median, maximum and minimum values, quartiles, and the two-sided 95\% confidence intervals (CIs) of the mean, were calculated for all efficacy endpoints and the additional endpoints. Analysis of covariance (ANCOVA) was used to analyze between-group differences in the change from baseline (week 0) in DTR-QOL total score (primary endpoint), total score for each factor of the DTR-QOL, and DTSQ total score at week 12 (end of study). The ANCOVA compared the treatment groups using the change from baseline in the score at the end of treatment [week 12 (end of study)] as a dependent variable, the DTR-QOL score at the start of the screening period $(<80$ or $\geq 80)$ and $\mathrm{HbA1c}$ $(<8.0 \%$ or $\geq 8.0 \%)$ at the start of the screening period as covariates, and treatment group as an independent variable. The two-sided level of significance was defined as 5\%. The week 12 (end of study) analysis accounted for those patients who withdrew from the study, while the week 12 analysis included only those who completed the study.

AEs were coded using the Medical Dictionary for Regulatory Activities (MedDRA) version 20.0. The incidences of AEs were calculated and presented by System Organ Class (SOC) and preferred term for each treatment group.

\section{RESULTS}

\section{Study Disposition and Patient Characteristics}

A total of 248 patients were screened for eligibility and provided informed consent. Of these, 219 were eligible for randomization, and 110 patients were randomized to receive trelagliptin, while 109 patients were randomized to daily DPP-4 inhibitor treatment. One patient in the daily DPP-4 inhibitor group had a protocol deviation and did not receive study treatment, which resulted in the FAS containing 
110 and 108 patients in the trelagliptin and daily DPP-4 inhibitor groups, respectively. All 110 patients in the trelagliptin group completed the study, while 105 patients in the daily DPP-4 inhibitor group completed the study. A total of two patients in the DPP-4 inhibitor group voluntarily withdrew from the study: one patient experienced an AE (urticaria) and one patient withdrew due to difficulties with visiting the hospital.

The demographic and baseline characteristics of the FAS are presented in Table 1. The total population $(N=218)$ had a mean age of 59.9 years and was $77 \%$ male. The mean duration of diabetes was 6.42 years, with $>77 \%$ of patients having diabetes for $<10$ years. At screening, mean HbA1c was $7.37 \%$ and $83.5 \%$ of patients had HbA1c $<8.0 \%$. Mean total score for the DTR-QOL at screening was 66.39, and $81.2 \%$ of the patient population had a DTRQOL total score $<80$.

Generally, the demographic and baseline characteristics were similar between the two groups (Table 1). Of note, $57.3 \%$ of patients in the trelagliptin group were aged $<65$ years, compared with $74.1 \%$ in the daily DPP- 4 inhibitor group.

Primary Efficacy Endpoint: Change from Baseline in DTR-QOL Total Score at Week 12 (End of Study)

The least-squares mean (LSM) change from baseline to week 12 (end of study) in DTR-QOL total score was 5.641 [standard error (SE) 1.859] and 3.223 (SE 1.871) in the trelagliptin and daily DPP-4 inhibitor groups, respectively. The between-group difference was 2.418 (95\% CI $-1.546,6.382 ; P=0.2305$ ) (Table 2).

\section{Secondary Efficacy Endpoints}

\section{Change from Baseline in Total Score for Each} DTR-QOL Factor at Week 12 (End of Study) The LSM change in total score from baseline to week 12 (end of study) was not significantly different between the two groups for any of the factors of the DTR-QOL (Table 2). Overall, the scores indicated that there were improvements from baseline in both groups in all factors over the study period.

\section{Change from Baseline in DTR-QOL and DTSQ Total Scores at Each Assessment Point}

The change from baseline in the DTR-QOL total score is shown in Fig. 1a. The total score improved over time for both treatment groups. However, the initial improvement in DTR-QOL score from baseline to week 4 was almost double in the trelagliptin group compared with the daily DPP-4 inhibitor group (8.04 vs. 4.82).

The change from baseline in total DTSQ score also demonstrated an improvement with treatment in both groups (Fig. 1b). The total score for the DTSQ at week 12 (end of study) showed a greater improvement for patients receiving trelagliptin than daily DPP-4 inhibitor recipients, but the difference was not significant (LSM 0.613; 95\% CI - 1.380, 2.605; $P=0.5451)$.

\section{Change from Baseline in DTR-QOL and DTSQ Total Scores Stratified by Medication Use}

The change from baseline in DTR-QOL and DTSQ total scores according to medication use is summarized in Table S1 and described in the Appendix in the electronic supplementary material. Overall, these analyses showed that the scoring was affected by medication use. Irrespective of treatment allocation, patients receiving fewer concurrent dose administrations and study drug administrations had the greatest improvements in DTR-QOL and DTSQ total scores.

\section{Additional Endpoints}

Overall, there were similar improvements in HbA1c, fasting blood glucose, fasting glycoalbumin, and 1,5-AG from baseline in both groups. At study end, the mean HbA1c decrease was $-0.48 \%$ and $-0.49 \%$ for the trelagliptin and daily DPP-4 inhibitor groups, respectively, while the mean fasting blood glucose decrease was $-7.2 \mathrm{mg} / \mathrm{dL}$ and $-12.7 \mathrm{mg} / \mathrm{dL}$. The mean change from baseline to study end in fasting glycoalbumin levels was $-1.49 \%$ in patients receiving trelagliptin and $-1.5 \%$ in those receiving daily DPP-4 inhibitors, and the mean 
Table 1 Demographics and baseline characteristics

\begin{tabular}{|c|c|c|}
\hline & $\begin{array}{l}\text { Trelagliptin } \\
100 \mathrm{mg} \\
(N=110)\end{array}$ & $\begin{array}{l}\text { Daily DPP-4 } \\
\text { inhibitors } \\
(N=108)\end{array}$ \\
\hline \multicolumn{3}{|l|}{ Age (years) } \\
\hline Mean (SD) & $61.5(8.96)$ & $58.4(10.01)$ \\
\hline \multicolumn{3}{|l|}{ Age categories $(N, \%)$} \\
\hline$<65$ years & $63(57.3)$ & $80(74.1)$ \\
\hline$\geq 65$ years & $47(42.7)$ & $28(25.9)$ \\
\hline \multicolumn{3}{|l|}{ Gender $(N, \%)$} \\
\hline Male & $86(78.2)$ & $82(75.9)$ \\
\hline \multicolumn{3}{|l|}{ BMI $\left(\mathrm{kg} / \mathrm{m}^{2}\right)$} \\
\hline Mean (SD) & $24.72(3.224)$ & $25.53(4.349)$ \\
\hline \multicolumn{3}{|c|}{ Smoking classification $(N, \%)$} \\
\hline Never smoked & $48(43.6)$ & $40(37.0)$ \\
\hline Current smoker & $24(21.8)$ & $28(25.9)$ \\
\hline Ex-smoker & $38(34.5)$ & $40(37.0)$ \\
\hline \multicolumn{3}{|c|}{ Drink alcohol almost every day? $(N, \%)$} \\
\hline Yes & $38(34.5)$ & $36(33.3)$ \\
\hline \multicolumn{3}{|c|}{ Duration of DM (years) } \\
\hline Mean (SD) & $6.45(4.972)$ & $6.39(5.286)$ \\
\hline \multicolumn{3}{|c|}{ Duration of DM categories $(N, \%)$} \\
\hline$<5$ years & $48(43.6)$ & $58(53.7)$ \\
\hline$\geq 5$ to $<10$ years & $37(33.6)$ & $26(24.1)$ \\
\hline$\geq 10$ years & $25(22.7)$ & $24(22.2)$ \\
\hline \multicolumn{3}{|c|}{ Any medication for concurrent condition $(N, \%)$} \\
\hline Yes & $50(45.5)$ & $41(38.0)$ \\
\hline$<2$ times & $46(92.0)$ & $39(95.1)$ \\
\hline$\geq 2$ times & $4(8.0)$ & $2(4.9)$ \\
\hline$<2$ tablets & $44(88.0)$ & $38(92.7)$ \\
\hline$\geq 2$ tablets & $6(12.0)$ & $3(7.3)$ \\
\hline \multicolumn{3}{|c|}{ Number of doses of study drugs $(N, \%)$} \\
\hline Once a week & $110(100.0)$ & $0(0.0)$ \\
\hline Once daily & $0(0.0)$ & $100(92.6)$ \\
\hline Twice daily & $0(0.0)$ & $8(7.4)$ \\
\hline
\end{tabular}

Table 1 continued

\begin{tabular}{lll}
\hline Trelagliptin & Daily DPP-4 \\
$100 \mathrm{mg}$ & inhibitors \\
& $(N=110)$ & $(N=108)$ \\
\hline
\end{tabular}

DTR-QOL total score

\begin{tabular}{|c|c|c|}
\hline Mean (SD) & $68.48(17.158)$ & $68.86(16.240)$ \\
\hline \multicolumn{3}{|c|}{ DTSQ total score ${ }^{a}$} \\
\hline Mean (SD) & $22.31(5.444)$ & $21.56(5.679)$ \\
\hline \multicolumn{3}{|l|}{ HbAlc (\%) } \\
\hline Mean (SD) & $7.40(0.819)$ & $7.34(0.620)$ \\
\hline
\end{tabular}

Fasting blood glucose $(\mathrm{mg} / \mathrm{dL})^{\mathrm{b}}$

$$
\text { Mean (SD) } \quad 150.0(27.85) \quad 151.3(30.67)
$$

Fasting glycoalbumin $(\%)^{\mathrm{b}}$

$$
\text { Mean (SD) } \quad 19.88(3.455) \quad 19.27(2.936)
$$

$1,5-\mathrm{AG}(\mu \mathrm{g} / \mathrm{mL})^{\mathrm{b}}$

\begin{tabular}{lll} 
Mean $(S D)$ & $7.03(4.796)$ & $7.38(4.756)$ \\
\hline
\end{tabular}

1,5- $A G$ 1,5-anhydroglucitol, 8-OHdG urinary 8-hydroxy2 -deoxyguanosine, $B M I$ body mass index, $D M$ diabetes mellitus, DPP-4 dipeptidyl peptidase 4, DTR-QOL Diabetes Therapy-Related Quality Of Life, DTSQ Diabetes Treatment Satisfaction Questionnaire, $H b A l c$ glycosylated hemoglobin A1c, $S D$ standard deviation

a Trelagliptin $N=108$; daily DPP-4 inhibitor $N=108$

b Trelagliptin $N=108$; daily DPP-4 inhibitor $N=107$

c Trelagliptin $N=99$; daily DPP-4 inhibitor $N=103$

change from baseline to study end in 1,5-AG was $2.74 \mu \mathrm{g} / \mathrm{mL}$ and $3.46 \mu \mathrm{g} / \mathrm{mL}$ in the trelagliptin group and the daily DPP-4 inhibitor group, respectively.

Mean study drug adherence was similar between groups, with $99.6 \%$ and $97.0 \%$ of patients in the trelagliptin and daily DPP-4 inhibitor group adherent to their medication, respectively (Table S2; electronic supplementary material). Although the adherence rate in both groups was high, there were some differences in the range of adherence seen, suggesting that patients in the daily DPP-4 inhibitor group missed doses more frequently than in the trelagliptin group (Table S2). The individual dosing time data are presented in Fig. S1 (electronic supplementary material), where the $Y$ axis 
Table 2 Changes in total score for each factor in the DTR-QOL questionnaire

\begin{tabular}{|c|c|c|c|c|}
\hline \multirow[t]{2}{*}{ Total score/factor } & \multicolumn{2}{|c|}{$\begin{array}{l}\text { Change from baseline to end of study } \\
\text { LSM (SE) }\end{array}$} & \multirow[t]{2}{*}{$\begin{array}{l}\text { Between-group difference } \\
\text { LSM (95\% CI) }\end{array}$} & \multirow[t]{2}{*}{$P$ value } \\
\hline & $\begin{array}{l}\text { Trelagliptin } \\
100 \mathrm{mg}\end{array}$ & $\begin{array}{l}\text { Daily DPP-4 } \\
\text { inhibitor }\end{array}$ & & \\
\hline Total score & $5.641(1.859)$ & $3.223(1.871)$ & $2.418(-1.546,6.382)$ & 0.2305 \\
\hline $\begin{array}{l}\text { Factor 1: burden on social activities } \\
\text { and daily activities }\end{array}$ & $6.385(2.386)$ & $4.447(2.402)$ & $1.938(-3.150,7.027)$ & 0.4536 \\
\hline $\begin{array}{l}\text { Factor 2: anxiety and dissatisfaction } \\
\text { with treatment }\end{array}$ & $4.670(2.255)$ & $0.510(2.270)$ & $4.160(-0.649,8.968)$ & 0.0896 \\
\hline Factor 3: hypoglycemia & $3.136(2.760)$ & $3.699(2.778)$ & $-0.563(-6.448,5.323)$ & 0.8506 \\
\hline Factor 4: satisfaction with treatment & $7.198(2.680)$ & $4.502(2.697)$ & $2.696(-3.018,8.410)$ & 0.3533 \\
\hline
\end{tabular}

$C I$ confidence interval, DTR-QOL Diabetes Therapy-Related Quality Of Life, DPP-4 dipeptidyl peptidase 4, LSM leastsquares mean, $S E$ standard error

indicates the difference from the appropriate dosing time [i.e., $24 \mathrm{~h}$ after the previous dosing time for once-daily DPP-4 inhibitor recipients (Fig. S1a), and $168 \mathrm{~h}$ after the previous dosing time for trelagliptin recipients (Fig. S1b)]. The mean absolute difference from the appropriate dosing time was $2.0 \mathrm{~h}$ in the once-daily DPP-4 inhibitor group and $5.6 \mathrm{~h}$ in the trelagliptin group (Table S3; electronic supplementary material).

\section{Safety and Tolerability}

A similar proportion of patients in each group experienced a treatment-emergent $\mathrm{AE}$ (TEAE; Table 3). The majority of these were mild to moderate in severity, with no severe events reported. One patient in the daily DPP-4 inhibitor group discontinued the study because of an AE of urticaria. Three patients in the trelagliptin group experienced a total of eight drug-related TEAEs (palpitations, constipation, nausea, thirst, musculoskeletal stiffness, dizziness, dyspnea, and rash). Three patients in the DPP-4 inhibitor group experienced a drug-related TEAE (gastro-esophageal reflux disease or malaise, or urticaria).

No deaths or serious drug-related TEAEs were reported. The two non-drug-related SAEs reported in the trelagliptin group were vertigo and diverticulitis, occurring in one patient each (Table 3). Both SAEs resolved with appropriate drug therapy.

Regarding AEs by SOC, the most common (occurring in $>3 \%$ of any group) were infections and infestations (trelagliptin vs. daily DPP-4 inhibitors: $8.2 \%$ vs. $4.6 \%$ ), gastrointestinal disorders $(3.6 \%$ vs. $5.6 \%)$, and musculoskeletal and connective tissue disorders $(3.6 \%$ vs. $2.8 \%$ ). The only individual TEAE that occurred in more than 3\% of patients in either group was viral upper respiratory tract infection (3.6\% vs. $1.9 \%)$.

\section{DISCUSSION}

The results of this study showed that trelagliptin was as effective as daily DPP-4 inhibitors at improving QOL and treatment satisfaction in treatment-naïve patients with T2DM. Numerically greater improvements with trelagliptin versus daily DPP-4 inhibitors were observed in the LSM change from baseline to week 12 (end of study) in the DTR-QOL total score, and in the individual factor (with the exception of hypoglycemia) and stratified scores for the DTR-QOL. However, no statistically significant differences were observed between the two treatment groups for any comparison of LSM changes from baseline to week 12 . 

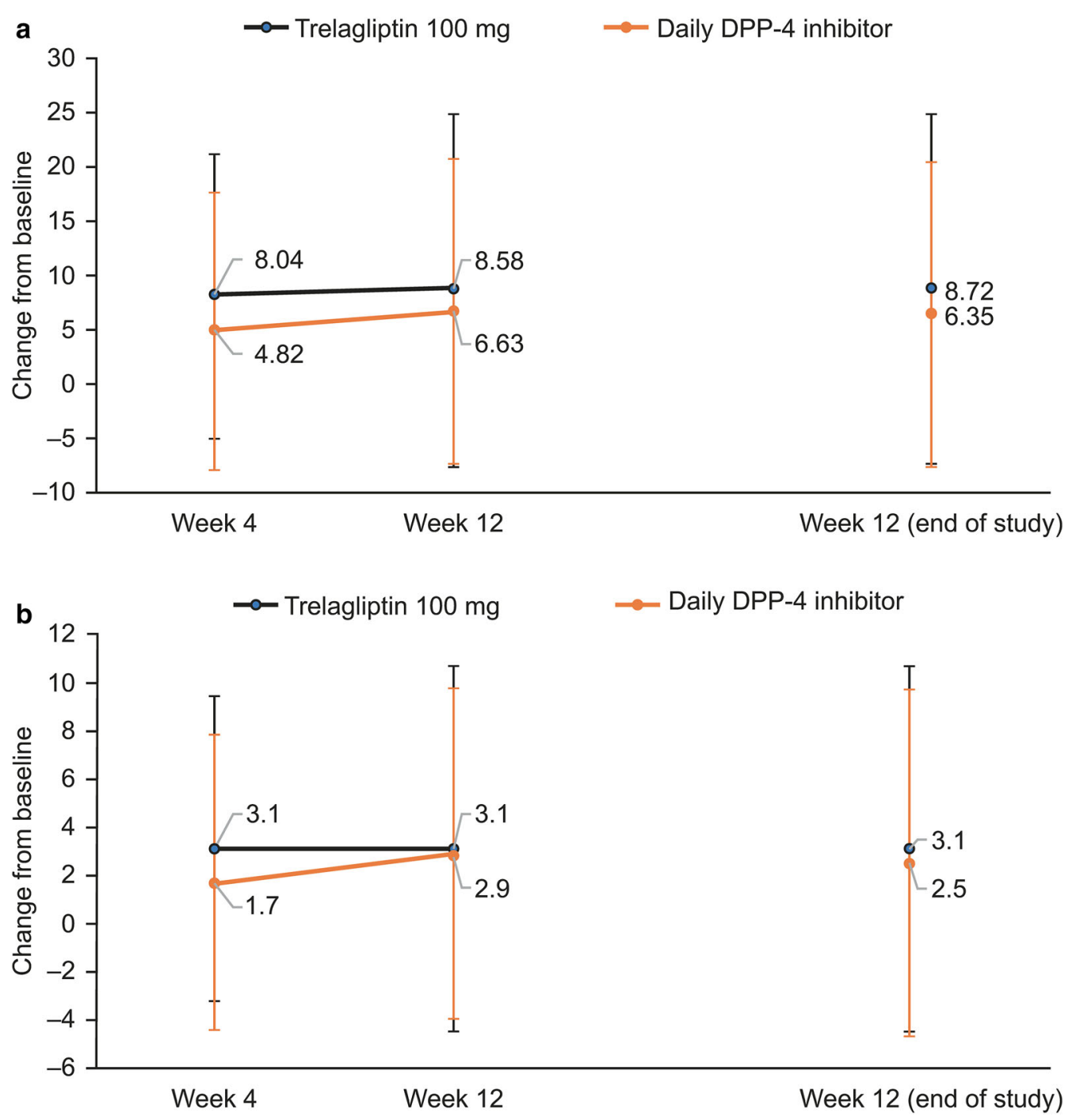

Fig. 1 Change from baseline at each assessment point in a DTR-QOL and b DTSQ. DTR-QOL Diabetes Therapy-Related Quality Of Life, DTSQ Diabetes Treatment Satisfaction Questionnaire

Improvements in DTSQ total score were also numerically greater in the trelagliptin group than the daily DPP-4 inhibitor group at each assessment point and at the end of the study; however, again, no statistical differences in DTSQ total score were observed between the two groups in the LSM change from baseline to week 12.

A study in 49 Japanese patients with T2DM who had received daily DPP-4 inhibitors for 3 months and were then continued on daily treatment or switched to trelagliptin for 12 weeks found that the change in DTR-QOL total score significantly improved with trelagliptin versus daily DPP-4 inhibitor treatment, and the subscale analysis also showed significantly greater changes with trelagliptin versus daily DPP-4 inhibitor treatment in domain 1 (burden of social activities/personal activities) and domain 2 (anxiety and dissatisfaction with treatment) [23]. Similar to the present study, there was no significant difference in the change in DTSQ total score between groups [23]. A potential reason why the results of this study contrasted with the aforementioned study in terms of the DTR-QOL result is that the 
Table 3 Overview of treatment-emergent adverse events

\begin{tabular}{llc}
\hline & \multicolumn{2}{l}{ Number of patients (\%) } \\
\cline { 2 - 3 } & $\begin{array}{l}\text { Trelagliptin } \\
\mathbf{1 0 0} \mathbf{~ m g} \\
(\boldsymbol{N}=\mathbf{1 1 0})\end{array}$ & $\begin{array}{c}\text { Daily DPP-4 } \\
\text { inhibitors } \\
(\boldsymbol{N}=\mathbf{1 0 8})\end{array}$ \\
\hline TEAEs & $19(17.3)$ & $20(18.5)$ \\
Mild & $16(14.5)$ & $17(15.7)$ \\
Moderate & $3(2.7)$ & $3(2.8)$ \\
Severe & $0(0.0)$ & $0(0.0)$ \\
Leading to & $0(0.0)$ & $1(0.9)$ \\
discontinuation & $3(2.7)$ & $3(2.8)$ \\
Drug-related TEAEs & $16(14.5)$ & $17(15.7)$ \\
Non-drug-related & & $0(0.0)$ \\
TEAEs & $2(1.8)$ & $0(0.0)$ \\
Serious TEAEs & $0(0.0)$ & $0(0.0)$ \\
Related & $2(1.8)$ & $0(0.0)$ \\
Not related & $0(0.0)$ & $0(0.0)$ \\
Leading to & & \\
discontinuation & $0(0.0)$ & \\
Deaths & &
\end{tabular}

$D P P-4$ dipeptidyl peptidase 4, TEAE treatment-emergent adverse event

patients in the present study were treatment naïve, and thus had no previous regimen for comparison, while the patients in the aforementioned study had been taking daily DPP-4 inhibitors for 3 months, which allowed them to fully experience both the daily and weekly regimens. Another study of 80 Japanese patients with T2DM found that a switch from daily to weekly DPP-4 inhibitors had no impact on DTSQ total score. However, a significant change in DTSQ total score from baseline was reported in patients who were treatment naïve [24]. The results of the aforementioned studies suggest that treatment with daily DPP-4 inhibitors increased treatment satisfaction from baseline and reduced the potential difference in satisfaction between daily and once-weekly DPP-4 inhibitors.

Evidence suggests that despite less frequent dosing, trelagliptin has comparable efficacy to daily DPP-4 inhibitors with respect to reducing HbA1c levels and other markers of glycemic control. Trelagliptin was found to be non-inferior to the once-daily DPP-4 inhibitor alogliptin in a phase III, randomized, double-blind study of 243 Japanese patients with T2DM [15], with both trelagliptin and alogliptin recipients demonstrating significant reductions in HbA1c versus placebo at the end of treatment. A study of 14 Japanese patients with T2DM who had stable glycemic control on sitagliptin and were switched to trelagliptin showed that blood glucose levels were either maintained or decreased after the switch to trelagliptin [25], suggesting that patients switched to trelagliptin do not experience deterioration in glycemic control. The results of these and other studies [23, 24] support the results of the present study, which showed that trelagliptin was as effective at improving parameters of glycemic control as daily DPP-4 inhibitor treatment.

Treatment adherence was high in this study, which may be a consequence of the enrolled patients being treatment naïve, and thus potentially more likely to be adherent to a new regimen in a clinical trial setting than what would occur in the real world. Of note, while the overall adherence rates between groups was similar, there was a lower 'quality' of adherence in the daily DPP-4 inhibitor group than in the trelagliptin group, with more missed doses in the daily DPP-4 inhibitor group than the weekly trelagliptin group, and a higher degree of variation in appropriate dosing time with daily DPP-4 inhibitors than with trelagliptin, considering the different dosing intervals [difference from the appropriate dosing time (SD): 2.0 (9.1) from $24 \mathrm{~h}$ vs. 5.6 (16.2) from $168 \mathrm{~h}$ ]. This variation is notable because differences in dose timing can lead to adverse outcomes, such as an increased risk of hypo- or hyperglycemia in patients with diabetes who require medication for glycemic control. So, while adherence was very good overall in both treatment groups, when dose timing was taken into account, adherence in the trelagliptin group was closer to the ideal administration situation than adherence in the daily DPP-4 inhibitor group. This may be due to the instructions provided in the drug package inserts in which the dosing is 
described as 'one time each day', e.g. for alogliptin, whereas trelagliptin dosing is described as 'once a week on the same day of the week'. Further details about the ideal dosing may be warranted in the drug package inserts.

Overall, these results suggest that a reduction in the frequency of dosing improves adherence. Other studies of patients with T2DM have shown that a reduction in dosing frequency increased adherence $[9,10]$, and surveys of patients with T2DM reported that reduction in dosing frequency was an important factor for patients, with some patients expressing a preference for once-weekly dosing over once-daily dosing [26]. In the present study, there was an improvement in DTR-QOL scores in patients receiving fewer concurrent dose administrations and study drug administrations, suggesting QOL was improved by once-weekly dosing in these patients, although due to the low number of patients included in the medication use analyses, these results should be interpreted with caution. However, taken together with the results of previous studies, these results suggest that the use of trelagliptin is likely to improve adherence in the long term, and may be a useful treatment option for patients who are not compliant with daily therapy.

Safety was similar between groups in the present study, with no new safety signals identified. Trelagliptin has a similar safety profile to daily DPP-4 inhibitors, as supported by other studies $[15,25]$.

This study has some limitations, including that the patients were treatment naïve before the start of the study, and that the study was conducted in a clinical trial setting, which may have resulted in patients being more adherent than what would have occurred in a real-world setting, leading to improvements in QOL and satisfaction for both treatments.

Given the similar efficacy and favorable safety and tolerability profile between onceweekly trelagliptin $100 \mathrm{mg}$ and daily DPP-4 inhibitor treatment in this study, the choice between the two drugs for treatment-naïve patients will depend on patient preference and lifestyle, as well as the treatment policy of physicians (which is likely to account for factors such as baseline glycemic control, diabetes duration, comorbidities and cardiovascular risk factors, and likelihood of adherence). The comprehension and co-operation of the patient are indispensable for the management and treatment of disease, particularly in diseases that are related to lifestyle. It is equally important to ensure that patients are receiving accurate and comprehensive information from their physicians so that patients together with their physicians are able to make informed and appropriate treatment choices. Moreover, clinical inertia, defined as the failure of healthcare providers to initiate or intensify therapy when indicated, is gaining prominent attention in diabetes therapy [27]. A treating physician could consider changing the treatment method in order to avoid clinical inertia upon recognizing a loss of adherence and/or treatment discontinuation in patients. For example, DPP-4 inhibitor treatment could be switched from daily to once weekly or vice versa.

\section{CONCLUSIONS}

Over 12 weeks of treatment, treatment-naïve patients with T2DM receiving once-weekly trelagliptin $100 \mathrm{mg}$ had a numerically greater, but not statistically greater improvement in QOL and treatment satisfaction compared with those receiving daily DPP-4 inhibitor treatment. Treatment efficacy in terms of glycemic control was similar with trelagliptin versus daily DPP-4 inhibitors, as was safety. In the absence of differences in efficacy and safety, the decision to administer once-weekly or daily DPP-4 inhibitor treatment is likely to depend on patient preferences and the treatment policy of physicians.

\section{ACKNOWLEDGEMENTS}

We thank the participants of the study, the investigators, and all study center staff at each site. The authors would like to acknowledge Prof. Clare Bradley and Health Psychology Research Limited as the owners of the DTSQ questionnaire, and to thank them for permitting its use in this trial. 
Funding. This study, the manuscript preparation, and article processing charges were sponsored by Takeda Pharmaceutical Company Ltd. All authors had full access to all of the data in this study and take complete responsibility for the integrity of the data and accuracy of the data analysis.

Medical Writing Assistance. Sheridan Henness, PhD, assisted with writing the first draft of this manuscript under the direction of the authors, on behalf of FireKite, an Ashfield company, part of UDG Healthcare plc, which was utilized during the development of this manuscript and was funded by Takeda Pharmaceutical Company Ltd., in compliance with Good Publication Practice 3 ethical guidelines (Battisti et al., Ann Intern Med 2015;163:461-4).

Authorship. All named authors meet the International Committee of Medical Journal Editors (ICMJE) criteria for authorship for this article, take responsibility for the integrity of the work as a whole, and have given their approval for this version to be published.

Authorship Contributions. Hitoshi Ishii contributed to the study design, and acquisition and interpretation of the data. Yuki Suzaki contributed to the study design and interpretation of the data. Yuko Miyata contributed to the study design and interpretation of the data. Shingo Matsui contributed to the acquisition and interpretation of the data. All authors critically reviewed, edited, and approved the final manuscript, and all authors agree to be accountable for all aspects of the work, which includes ensuring that questions related to the accuracy or integrity of any part of the work are appropriately investigated and resolved.

Disclosures. Hitoshi Ishii has received lecture and/or consultant fees from Daiichi Sankyo Company, Ltd., Eli Lilly Japan K.K., MSD K.K., Mitsubishi Tanabe Pharma Corporation, Novo Nordisk Pharma Ltd., Sanofi K.K., Shionogi \& Co. Ltd., Sumitomo Dainippon Pharma, and Takeda Pharmaceutical Company, Ltd. Yuki Suzaki is an employee of Takeda Pharmaceutical Company Limited.Yuko Miyata is an employee of Takeda Pharmaceutical Company Limited. Shingo Matsui is an employee of Takeda Pharmaceutical Company Limited.

Compliance with Ethics Guidelines. The study was conducted in compliance with the Japanese Ethical Guideline for Clinical Research, all applicable local regulations, and the ethical principles set out in the Declaration of Helsinki and the Good Clinical Practice consolidated guidelines. All regulation-specified documentation, including the study protocol and all revisions, were reviewed and approved by an independent ethics committee at each study site (as listed in the electronic supplementary material). All patients provided written informed consent before any screening procedures were undertaken.

Data Availability. The datasets generated during and/or analyzed during the current study are available at ClinicalStudyDataRequest.com: https://www.clinicalstudydatarequest. com/Default.aspx

Open Access. This article is distributed under the terms of the Creative Commons Attribution-NonCommercial 4.0 International License (http://creativecommons.org/licenses/ by-nc/4.0/), which permits any noncommercial use, distribution, and reproduction in any medium, provided you give appropriate credit to the original author(s) and the source, provide a link to the Creative Commons license, and indicate if changes were made.

\section{REFERENCES}

1. American Diabetes Association. Introduction: standards of medical care in diabetes-2018. Diabetes Care. 2018;41(Suppl 1):S1-2.

2. American Diabetes Association. Diagnosis and classification of diabetes mellitus. Diabetes Care. 2014;37(Suppl 1):S81-90.

3. Japan Diabetes Society. Treatment guide for Diabetes 2016-2017. 2016. http://www.fa.kyorin.co.jp/ jds/uploads/Treatment_Guide_for_Diabetes_20162017.pdf. Accessed Feb 5, 2019. 
4. Lu Y, Wang N, Chen Y, et al. Health-related quality of life in type-2 diabetes patients: a cross-sectional study in East China. BMC Endocr Disord. 2017;17:38.

5. Safita N, Islam SM, Chow CK, et al. The impact of type 2 diabetes on health related quality of life in Bangladesh: results from a matched study comparing treated cases with non-diabetic controls. Health Qual Life Outcomes. 2016;14:129.

6. Wandell PE. Quality of life of patients with diabetes mellitus. An overview of research in primary health care in the Nordic countries. Scand J Prim Health Care. 2005;23:68-74.

7. Al-Khaledi M, Al-Dousari H, Al-Dhufairi S, et al. Diabetes self-management: a key to better healthrelated quality of life in patients with diabetes. Med Princ Pract. 2018;27:323-31.

8. Garcia-Perez LE, Alvarez M, Dilla T, Gil-Guillen V, Orozco-Beltran D. Adherence to therapies in patients with type 2 diabetes. Diabetes Ther. 2013;4:175-94.

9. Dezii CM, Kawabata H, Tran M. Effects of oncedaily and twice-daily dosing on adherence with prescribed glipizide oral therapy for type 2 diabetes. S Med J. 2002;95:68-71.

10. Kardas P. The DIACOM study (effect of DosIng frequency of oral Antidiabetic agents on the COMpliance and biochemical control of type 2 diabetes). Diabetes Obes Metab. 2005;7:722-8.

11. Saini SD, Schoenfeld P, Kaulback K, Dubinsky MC. Effect of medication dosing frequency on adherence in chronic diseases. Am J Manag Care. 2009;15:e22-33.

12. Paes $\mathrm{AH}$, Bakker A, Soe-Agnie CJ. Impact of dosage frequency on patient compliance. Diabetes Care. 1997;20:1512-7.

13. Patel BD, Ghate MD. Recent approaches to medicinal chemistry and therapeutic potential of dipeptidyl peptidase-4 (DPP-4) inhibitors. Eur J Med Chem. 2014;74:574-605.

14. Saisho Y. Alogliptin benzoate for management of type 2 diabetes. Vasc Health Risk Manag. 2015;11:229-43.

15. Inagaki $\mathrm{N}$, Onouchi $\mathrm{H}$, Maezawa $\mathrm{H}$, Kuroda $\mathrm{S}$, Kaku K. Once-weekly trelagliptin versus daily alogliptin in Japanese patients with type 2 diabetes: a randomised, double-blind, phase 3, non-inferiority study. Lancet Diabetes Endocrinol. 2015;3:191-7.

16. Kaku K. First novel once-weekly DPP-4 inhibitor, trelagliptin, for the treatment of type 2 diabetes mellitus. Expert Opin Pharmacother. 2015;16: 2539-47.

17. Ishii H, Suzaki Y, Miyata Y. Effect of trelagliptin on quality of life in patients with type 2 diabetes mellitus: study protocol. Diabetes Ther. 2017;8:1415-25.

18. Ishii H. Development and psychometric validation of the Diabetes Therapy-Related QOL (DTR-QOL) questionnaire. J Med Econ. 2012;15:556-63.

19. Bradley C. Diabetes treatment satisfaction questionnaire. In: Bradley C, editor. Handbook of psychology and diabetes: a guide to psychological measurement in diabetes research and practice. Langhorne: Harwood Academic; 1994. p. 111-32.

20. Ishii H, Bradley C, Riazi A, Barendse S, Yamamoto T. The Japanese version of the Diabetes Treatment Satisfaction Questionnaire (DTSQ): translation and clinical evaluation [Japanese]. J Clin Exp Med. 2000;192:809-14.

21. Yamazaki M, Higo N, Kaneko T, et al. SGLT2 inhibitors as the trigger for diabetes care: reconfirmed importance of behaviour modification after drug administration. J Japan Diabetes Soc. 2015;58: $745-52$.

22. Izumi K, Noda M. In order to reduce the interruption of diabetes treatment [Japanese]. Diabetes Front. 2014;25:709-18.

23. Oita M, Miyoshi H, Ono K, et al. Satisfaction and efficacy of switching from daily dipeptidyl peptidase- 4 inhibitors to weekly trelagliptin in patients with type 2 diabetes -randomized controlled study. Endocr J. 2018;65:141-50.

24. Tosaki T, Kamiya H, Yamamoto Y, et al. Efficacy and patient satisfaction of the weekly DPP-4 inhibitors trelagliptin and omarigliptin in 80 Japanese patients with type 2 diabetes. Intern Med. 2017;56:2563-9.

25. Inagaki N, Sano H, Seki Y, Kuroda S, Kaku K. Efficacy and safety of once-weekly oral trelagliptin switched from once-daily dipeptidyl peptidase- 4 inhibitor in patients with type 2 diabetes mellitus: An open-label, phase 3 exploratory study. J Diabetes Investig. 2018;9:354-9.

26. Hauber AB, Tunceli K, Yang JC, et al. A survey of patient preferences for oral antihyperglycemic therapy in patients with type 2 diabetes mellitus. Diabetes Ther. 2015;6:75-84.

27. Reach G, Pechtner V, Gentilella R, Corcos A, Ceriello A. Clinical inertia and its impact on treatment intensification in people with type 2 diabetes mellitus. Diabetes Metab. 2017;43:501-11. 